\title{
The ANTARES cosmic-neutrino detector in the Mediterranean: a status report
}

\author{
Vincenzo Flaminio for the ANTARES Collaboration* \\ Physics Department, University of Pisa and INFN Sez. di Pisa, Largo B. Pontecorvo, 56127 \\ Pisa, Italy \\ E-mail: vincenzo.flaminiodcern.ch
}

\begin{abstract}
The detection of very high energy neutrinos of galactic/extragalactic origin requires a very large detector mass and a large overburden as a shield against cosmic ray muons. Experiments are therefore being built under huge layers of ice or water, using the solid/liquid medium itself as a Cherenkov radiator.

ANTARES is the largest experiment of this kind currently under construction in the northern hemisphere. It is being built and installed at a depth of $2500 \mathrm{~m}$ in the Mediterranean sea, near the Southern French coast, by a large European collaboration. The experiment aims at the detection of high-energy neutrinos from astrophysical sources such as quasars, microquasars, supernova remnants, GRBs and others. A three-dimensional array of photomultipliers will be used to detect the Cherenkov light emitted by neutrino-induced muons. Thanks to the long scattering length of light in seawater and the excellent time resolution of the detector components, a very good muon/shower angular resolution is achieved, implying a good angular resolution for high energy neutrinos as well. The array consists of 12 lines each covering a vertical length of about $480 \mathrm{~m}$ of which $350 \mathrm{~m}$ are equipped with 75 photomultipliers arranged in triplets. The complete array, comprising 900 photomultipliers and the readout electronics, is connected to an on-shore laboratory through a $42 \mathrm{~km}$ long electro-optical cable. Prototype lines have been installed and operated over the last few years. Based on the experience acquired, the final detector design has been completed and a new instrumented line (MILOM) including 4 PMTs in addition to devices for monitoring environmental parameters, has been installed. Data taking is now underway, while the installation of the full detector will begin in January 2006 and will be completed in 2007. Details of the construction and installation, together with preliminary results obtained are presented.
\end{abstract}

International Europhysics Conference on High Energy Physics

July 21st - 27th 2005

Lisboa, Portugal

${ }^{*}$ Speaker. 


\section{The Antares detector}

ANTARES is a multidisciplinary experiment, whose main aim, of detecting neutrinos of cosmic origin, is accompanied by parallel research interests in the fields of marine biology and geophysics [1], [2], [3].

The detector consists of 12 lines, each holding 75 10" Hamamatsu photomultipliers (PMT) arranged in triplets (storeys) and looking downward, at an angle of $45^{\circ}$ to the vertical. The PMTs are housed in pressure-resistent glass spheres. The separation between storeys in each line is $14.5 \mathrm{~m}$, starting $100 \mathrm{~m}$ from the sea-floor; the distance between pairs of strings in the horizontal plane is $60-70 \mathrm{~m}$. Cherenkov light from muons originating from neutrino interactions in the Earth's crust underneath the detector is registered by the PMT array. Each PMT is read-out by Analog Ring Sampler ASICs, recording time and pulse-height information. The complete waveform may also be recorded, at a sampling rate of $1 \mathrm{GHz}$, for signals above a preset amplitude. Each PMT triplet is held in place by a titanium frame, attached to a vertical electro-optical cable used for data and clock signals as well as for power transmission. Digital data transmission uses optical fibres. At the center of the frame a titanium cylinder encloses the readout/control electronics, together with compasses/tiltmeters used for geometrical positioning. Some of the storeys also house LED beacons, each containing 60 LEDs, which provide very fast pulses used for timing calibrations.

A laser (laser beacon) located at the bottom of each line, provides additional means for timing calibrations. Hydrophones attached to each storey are used, in conjunction with sonic transmitters located at fixed locations on the sea-floor, for precise position determinations.

Each line is connected, via an electronics module located at its bottom (String Control Module - SCM), to a junction box (JB) in turn connected via a $42 \mathrm{~km}$ long electro-optical cable (installed in November 2001) to the shore station. All data are here collected by a computer farm, where a fast processing of events satisfying predetermined trigger requirements is performed. Precise timing is provided by a $20 \mathrm{MHz}$ high accuracy on-shore clock synchronised with the GPS, distributed via the electro-optical cable and the JB to each electronics module.

Since 2001 measurements have been made, using preliminary and prototype versions of our setup, at or close the chosen site [4], [5], [6]. As an example we show in figure 1(a), the angular distribution of muons measured in 2001, using a prototype sector line containing 7 PMTs.

The results of further tests made in 2003 showed some problems that were then corrected, leading to the final mechanical and electronic design. A new prototype line (MILOM), including just 4 PMTs in addition to several calibration/monitoring instruments and using the final design, has been installed and connected to the JB in March 2005.

\section{MILOM setup and performance}

A sketch of the MILOM is shown in figure 1(b). It has three storeys, in addition to the SCM and the Bottom String Socket (BSS) which contains, in addition to the acoustic release system, a seismometer, an acoustic position transducer (RxTx) and a laser beacon. Storey 1, located about $100 \mathrm{~m}$ above, has a LED beacon, and acoustic positioning hydrophone, a light transmission meter and a conductivity/temperature probe. Storey 2, $15 \mathrm{~m}$ above storey 1 , has three optical modules 


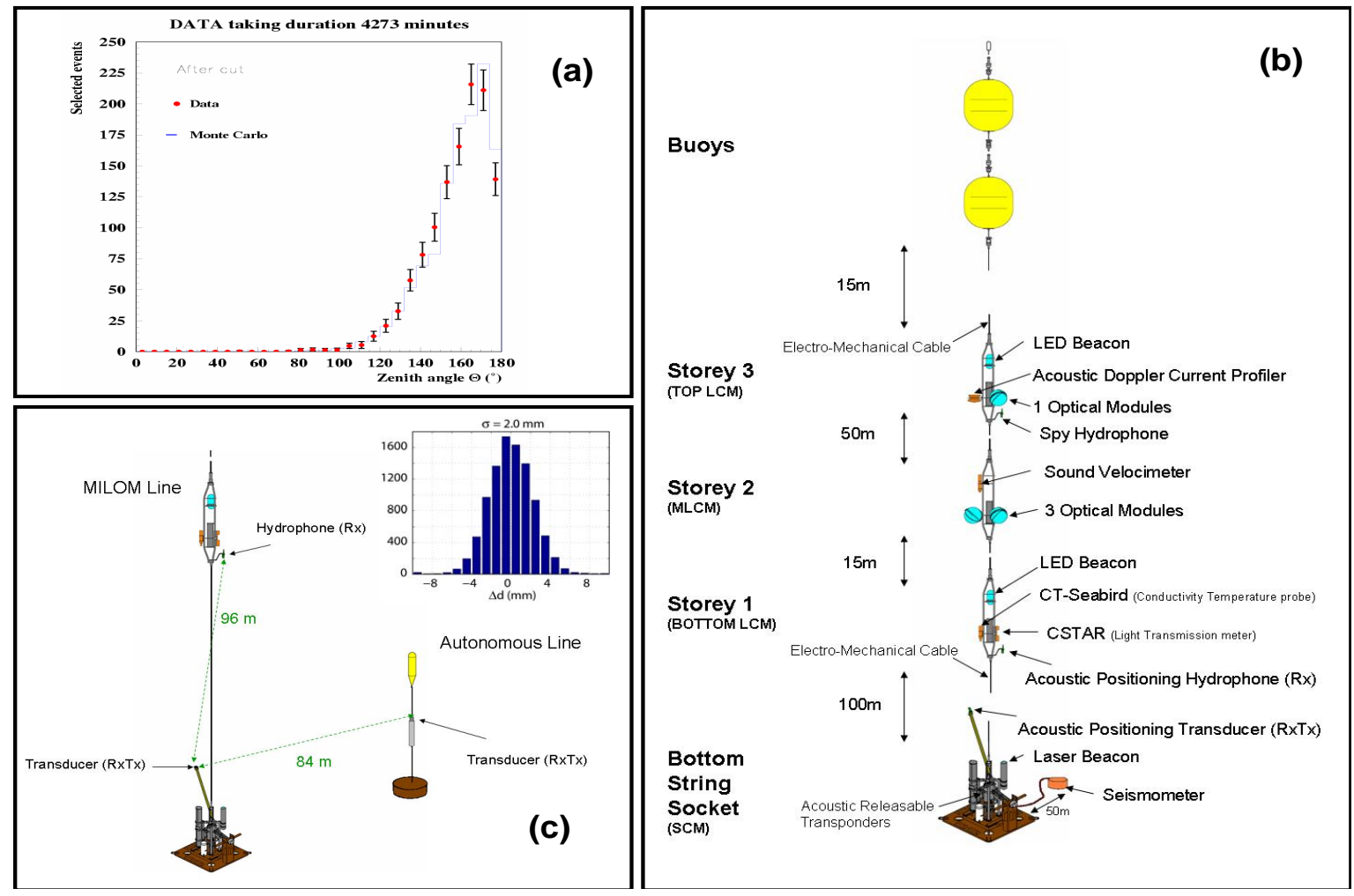

Figure 1: (a) Muon angular distribution measured in one of the early tests; (b) Sketch of the MILOM, showing all its components; (c) Setup used for testing the acoustic positioning system. The inset shows the distribution of vertical distance between the bottom of the MILOM and storey 1, measured by the hydrophones.

and a sound velocimeter. Storey 3,50 m above storey 2, has a hydrophone, one optical module, an acoustic doppler current profiler and a LED beacon. The MILOM is connected to the JB, which has been operational since its installation in December 2002. A large quantity of data has been acquired since the installation of the MILOM. In addition to regular data taking activity, mainly aimed at checking the background levels and their time variations, the following functionality tests have been performed:

1. Stability of the clock synchronisation signals over the $42 \mathrm{~km}$ distance between the shore station and the SCM. Over the first 42 days of operation we find time variations smaller than $1.5 \mathrm{~ns}$. These may be explained by changes in temperature affecting the part of the electro-optical cable laying in shallow waters. The clock delay between the SCM and storey 1 is stable within $50 \mathrm{ps}$.

2. Acoustic positioning resolution. This has been checked, for the time being, only in the onedimensional case. Use has been made of an autonomous line (shown in figure 1(c)) equipped with an acoustic transducer and placed $84 \mathrm{~m}$ away from the MILOM, in combination with the already mentioned hydrophones attached to the MILOM. The distribution of measured distances between the SCM and the autonomous line installed at a distance of $84 \mathrm{~m}$ has a $\sigma$ 
of $3.0 \mathrm{~cm}^{1}$. The spread in measured distances between the SCM and the hydrophone located on storey 1 , shown in the inset of figure 1 (c), has a $\sigma$ of $2 \mathrm{~mm}$. The design values in both instances are $10 \mathrm{~cm}$.

3. Optical Modules timing resolution. This is continuosusly monitored using the LED beacons. The light pulse from each beacon is precisely timed by a small PMT placed in front of it. The output from this PMT, shown in figure 2(a), has a $\sigma$ of 0.75 ns. Figure 2(b) shows the corresponding distribution of time differences between the signals recorded by pairs of PMTs of storey 2, located $15 \mathrm{~m}$ above. This distribution is centered at $0 \mathrm{~ns}$ and has a $\sigma$ of $0.75 \mathrm{~ns}$, implying a single PMT time resolution of $0.5 \mathrm{~ns}$. This resolution is for large pulses and is thus dominated by electronics effects rather than by the transit time spread of the PMT. The time delay between the LED pulse (measured by the small PMT) and the pulses from the detector PMTs has also been measured and the results compared with those of identical measurements made, before the installation, in the laboratory. The two sets of measurements agree within 2-3 ns, the difference being compatible with expectations from temperature differences in the two cases.

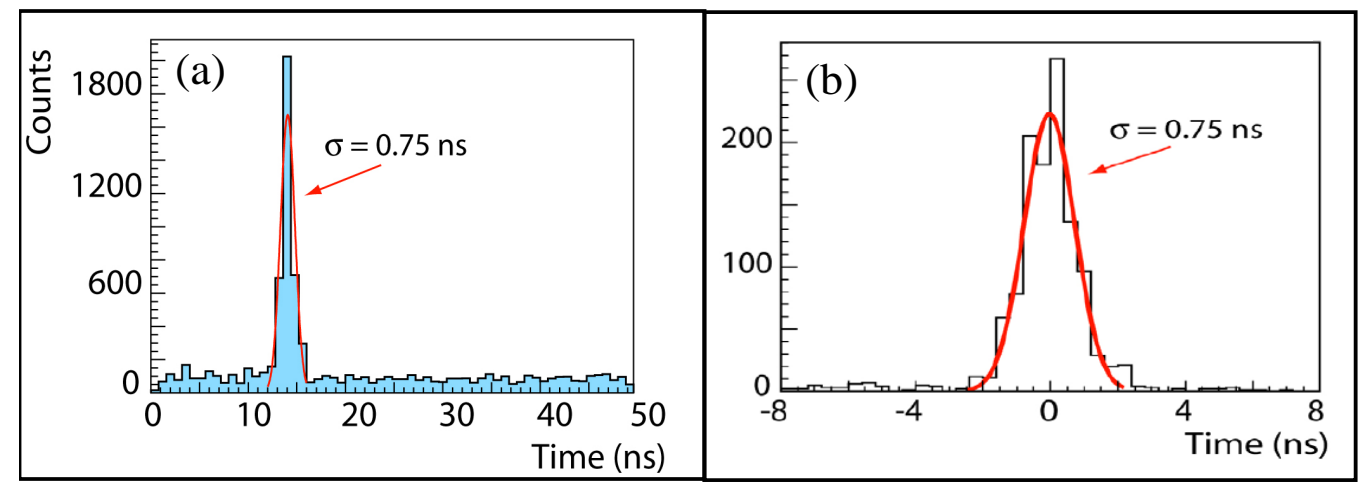

Figure 2: (a) Time distribution of signals from the small PMT placed in front of the LED beacon of storey 1; (b) Distribution of time differences between signals from pairs of PMTs of storey 2, when the LED beacon of storey 1 is pulsed.

4. Background rates. The 3 PMTs of storey 1 monitor in a continuous way the rate (mainly due to ${ }^{40} \mathrm{~K}$ and bioluminescence). With a PMT threshold set at 0.5 p.e., we find counting rates characterised by a baseline variable between 60 and $100 \mathrm{kHz}$, though periods with higher rates have been observed. Also sudden and short localised bursts reaching up to $1 \mathrm{MHz}$, due to living organisms have been observed. We find a correlation between the measured rate and the water current speed.

\section{Conclusions}

The successful deployment of the new instrumentation line (MILOM), also equipped with 4 standard ANTARES PMTs and readout electronics, has enabled us to test the performance of a

\footnotetext{
${ }^{1}$ This is mainly due to movements of the autonomous line. Recent measurements made using a new acoustic source, placed in a stable position at about $175 \mathrm{~m}$ from the MILOM, show a space resolution better than $1 \mathrm{~cm}$
} 
section of the ANTARES detector in its final configuration, while -at the same time- recording information on the various ambient parameters. We find that, after about three years since the installation of the $42 \mathrm{~km}$ long electro-optical cable and Junction Box, the system is fully functional. Tests of acoustic positioning give results well within specifications. Measurements of time response of the PMTs, made using fast LED pulses, show a time resolution of about $0.5 \mathrm{~ns}$ per PMT. The background counting rates, dominated by ${ }^{40} \mathrm{~K}$ decays and bioluminescence, are characterised by a baseline of $60 \div 100 \mathrm{kHz}$. Construction of the detector is now underway and is expected to be completed in 2007.

\section{References}

[1] "Proposal for a $0.1 \mathrm{~km}^{2}$ detector", the ANTARES Collaboration (1999), available at $\mathrm{http}: / /$ antares.in2p3.fr

[2] P. Amram et al., "The ANTARES optical module", Nuclear Instruments and Methods in Physics Research A484 (2002) 369

[3] J. A. Aguilar et al., "Study of Large Hemispherical Photomultiplier Tubes for the ANTARES Neutrino Telescope", Nuclear Instruments and Methods in Physics Research A 555 (2005) 132-141

[4] J.A. Aguilar et al., "Transmission of light in deep sea water at the site of the Antares neutrino telescope", Astroparticle Physics 23 (2005) 131-155 (astro-ph/0412126)

[5] P. Amram et al., "Sedimentation and Fouling of Optical Surfaces at the ANTARES Site", Astroparticle Physics 19 (2003) 253-267 (astro-ph/0206454)

[6] P. Amram et al., "Background light in potential sites for the ANTARES undersea neutrino telescope", Astroparticle Physics 13 (2000) 127-136 (astro-ph/9910170) 